\title{
Intramural Duodenal Hematoma In 2-year Old Child
}

\section{Dayanand Hota, ${ }^{*}$ Kamal Nain Rattan, Ahmad Khursheed, Manish Swami, Harish Bhardwaj}

Postgraduate Institute of Medical Sciences Rohtak, India

\section{ABSTRACT}

Background: Intramural hematoma of the duodenum is a rare cause of acquired duodenal obstruction. It is often a diagnostic dilemma and a high degree of suspicion is required to make an early and accurate diagnosis in children.

Case Report: A 2-year-old child presented with bilious vomiting and abdominal distension. X-ray abdomen showed high intestinal obstruction. Ultrasound and CT scan abdomen gave suspicion of pancreatic pseudo-cyst near head of pancreas. At surgery, an intramural hematoma of the duodenum was found and drained.

Conclusion: Intramural duodenal hematoma is a rare entity especially in children and should be considered in differential diagnosis of acquired duodenal obstruction.

Key words: Duodenal obstruction; Intramural duodenal hematoma; Bilious vomiting

Correspondence*: Dayanand Hota, Department of Pediatrics, PGIMS, Rohtak, India.

E-mail: dayanandhota@gmail.com @ (2018, Hota et al. .

Submitted: 24-01-2018

Accepted: 12-04-2018

Conflict of Interest: None

Source of Support: Nil

This is an open-access article distributed under the terms of the Creative Commons Attribution License, which permits unrestricted use, distribution, and reproduction in any medium, provided the original work is properly cited.

\section{INTRODUCTION}

Intramural duodenal hematoma most commonly occurs after trauma. It results in duodenal obstruction. There are other causes of intramural hematoma but are rare.

Duodenal injuries in children usually occur following blunt trauma and in most of the cases it also involves paraduodenal structures such as head of pancreas. Isolated duodenal involvement is quite rare.[1]

We are reporting a case of duodenal obstruction by a para-duodenal hematoma in a 2-year-old who presented with bilious vomiting.

\section{CASE REPORT}

A previously healthy 2-year-old boy admitted with complaint of bilious vomiting, abdominal distension, and excessive cry. On examination signs of mild dehydration were noted. Child was started on IV fluids, antibiotics and a nasogastric tube was placed.

Complete blood count showed mild anemia. Serum electrolytes and coagulation profile were within normal limits. X-ray abdomen showed high intestinal obstruction with double bubble sign and prominent gastric shadow. Ultrasonography showed a cystic lesion related to head of pancreas. Computed tomography showed a large cystic mass in relation to pancreas. No intralesional blood flow was observed on color Doppler.

A diagnosis of pseudocyst of pancreas was made (Figure 1). An exploratory laparotomy was performed which showed a large hematoma compressing 2nd and 3rd parts of the duodenum without involvement of surrounding structures. The serosa was opened and he- 
matoma was drained. Child was orally allowed after 72 hours. Patient is doing well on follow up.

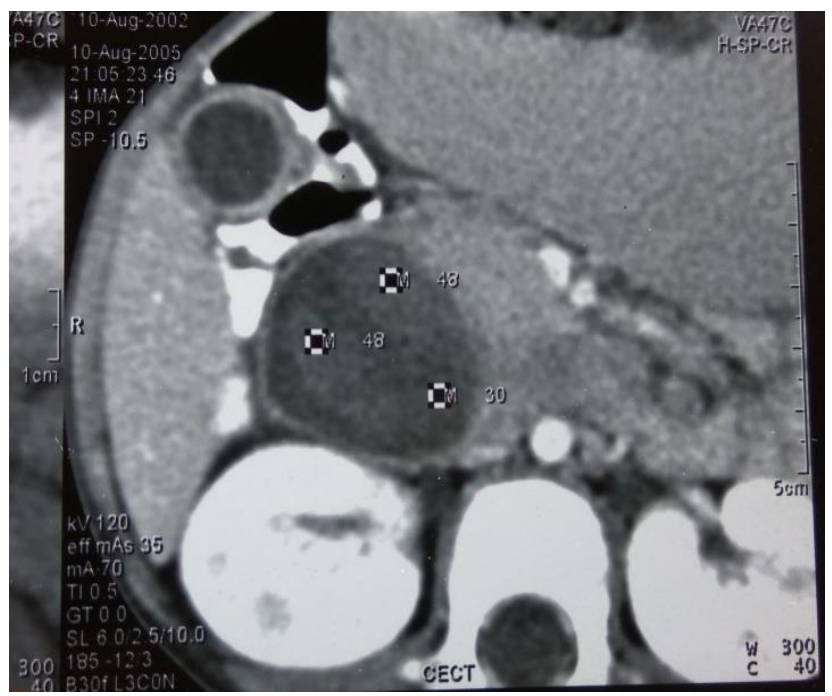

Figure 1: CECT abdomen showing a well-defined cystic mass in relation to head of pancreas

\section{DISCUSSION}

Intramural duodenal hematoma is rare in children and occurs only in $2-3 \%$ cases of blunt abdominal trauma. Anatomical factors which are thought to account for the higher incidence of duodenal involvement are rich submucosal vascular plexus in duodenum, immobile short duodenal mesentery, varying tensile strength of three layers of duodenal wall, lack of complete circumferential well developed serosal layer in the retroperitoneal portion of duodenum with resultant decreased ability to tamponade active intramural hemorrhage and the high flared costal margin common with less developed abdominal musculature.[2]

The proximity of pancreas to duodenum also increases the chance of traumatic pancreatitis with duodenal injury.[3-5] Occasionally trivial trauma may cause duodenal hematoma.[4] Trauma results in contusion and parietal bleeding which forms the hematoma. Such hematoma may grow and gradually cause luminal obstruction. Coagulopathies are also associated with such hematoma so related investigations should be carried out.[3-5]
In our case no coagulopathy was found as a cause of intramural duodenal hematoma. Differential diagnoses include duodenal duplication cyst, choledochal cyst, pancreatic pseudo-cyst etc. Double bubble sign was found in the index case. Ultrasound showed a cystic mass around head of pancreas. The conservative management consists of gastric decompression and parenteral nutrition.

Ultrasonographic follow up at regular interval should be done. Conservative treatment is successful and safe, particularly if diagnosis is made early.[5]. If neither clinical improvement nor resolution is observed within 2 weeks, or a perforation is suspected along with other associated injuries requiring surgical intervention, surgery is indicated in post traumatic intra mural duodenal hematoma.[2,5].

Consent: Authors declared that they have taken informed written consent, for publication of this report along with clinical photographs/material, from the legal guardian of the patient.

Authors' Contribution: All authors contributed equally in concept, literature review, and drafting of the manuscript. Both authors approved the final version of this manuscript.

\section{REFERENCES}

1. luchtman $M$, Steiner T, Faierman $T$, Breitgand $A$, Bartal G. Post-traumatic intramural duodenal hematoma in children. Isr Med Assoc J. 2006; 8:95-7.

2. Lam JPH, Eunson GJ, Munro FD, Orr JD. Delayed presentation of handlebar injuries in children. BMJ. 2001; 322:1288-9.

3. Stiekema J, Derikx JPM. Abdominal handlebar injury. JAMA Surg [Internet]. [cited 2017 Dec 20]; Available from:

https://jamanetwork.com/journals/jamasurgery/fullarticle /2664656

4. Desai KM, Dorward IG, Minkes RK, Dillon PA. Blunt duodenal injuries in children. J Trauma Acute Care Surg. 2003;54:640-6.

5. Peterson ML, Abbas PI, Fallon SC, Naik-Mathuria BJ, Rodriguez JR. Management of traumatic duodenal hematomas in children. J Surg Res. 2015;199:126-9. 